\title{
Article \\ Comprehensive Analysis of SFRP Family Members Prognostic Value and Immune Infiltration in Gastric Cancer
}

\author{
Dehua Liu ${ }^{1}(0)$, Chenyu Sun ${ }^{2}(0)$, Nahyun Kim ${ }^{2}{ }^{-}$, Chandur Bhan ${ }^{2}$, John Pocholo Whitaker Tuason ${ }^{2}$, Yue Chen ${ }^{3}$, \\ Shaodi Ma ${ }^{4}$, Yuting Huang ${ }^{5}\left(\mathbb{D}\right.$, Ce Cheng ${ }^{6,7}$, Qin Zhou ${ }^{8}$ and Kaiguang Zhang ${ }^{1, *(\mathbb{D})}$
}

check for

updates

Citation: Liu, D.; Sun, C.; Kim, N.; Bhan, C.; Tuason, J.P.W.; Chen, Y.; Ma, S.; Huang, Y.; Cheng, C.; Zhou, Q.; et al. Comprehensive Analysis of SFRP Family Members Prognostic Value and Immune Infiltration in Gastric Cancer. Life 2021, 11, 522. https://doi.org/10.3390/life11060522

Academic Editors: Barbara Illi and Lluís Ribas de Pouplana

Received: 21 April 2021

Accepted: 31 May 2021

Published: 3 June 2021

Publisher's Note: MDPI stays neutral with regard to jurisdictional claims in published maps and institutional affiliations.

Copyright: (c) 2021 by the authors. Licensee MDPI, Basel, Switzerland. This article is an open access article distributed under the terms and conditions of the Creative Commons Attribution (CC BY) license (https:/ / creativecommons.org/licenses/by/ $4.0 /)$.
1 The First Affiliated Hospital of USTC, Division of Life Sciences and Medicine, University of Science and Technology of China, Hefei 230001, China; ldh621@mail.ustc.edu.cn

2 Internal Medicine, AMITA Health Saint Joseph Hospital Chicago, Chicago, IL 60657, USA; chenyu.sun@amitahealth.org (C.S.); nahyun.kim@amitahealth.org (N.K.); Chandur.bhan@amitahealth.org (C.B.); johnpocholo.tuason@amitahealth.org (J.P.W.T.)

3 Department of Clinical Medicine, School of the First Clinical Medicine, Anhui Medical University, Hefei 230032, China; 1913010067@stu.ahmu.edu.cn

4 Department of Epidemiology and Health Statistics, School of Public Health Anhui Medical University, Hefei 230032, China; mashaodi@stu.ahmu.edu.cn

5 University of Maryland Medical Center Midtown Campus, Baltimore, MD 21201, USA; yuting.huang@umm.edu

6 The University of Arizona College of Medicine, Banner University Medical Center at South Campus, Tucson, AZ 85724, USA; ce.cheng@bannerhealth.com

7 Banner-University Medical Center South, Tucson, AZ 85713, USA

8 Radiation Oncology, Mayo Clinic, Rochester, MN 55905, USA; zhou.qin@mayo.edu

* Correspondence: zhangkaiguang@ustc.edu.cn; Tel.: +86-138-5517-0097

\begin{abstract}
Gastric cancer (GC) is the fifth most common cancer globally. Secreted frizzled-related proteins (SFRP) are important elements associated with the Wnt signaling pathway, and its dysregulated expression is found in multiple cancers. However, the function of distinct SFRPS in GC remains poorly understood. We investigated the differential expression, prognostic value, and immune cell infiltration of SFRPs in gastric cancer patients from the Oncomine, Gene Expression Profiling Interactive Analysis (GEPIA), UALCAN, Kaplan-Meier plotter, cBioPortal, STRING, Gene-MANIA, DAVID, MethSurv, and TIMER databases. We found that the expression levels of SFRP2 and SFRP4 were significantly increased in GC tissues, whereas the SFRP1 and SFRP5 expressions were reduced. $S F R P 1, S F R P 2$, and SFRP5 were significantly correlated with the clinical cancer stage in GC patients. Higher expression of SFRPS was associated with short overall survival (OS) in GC patients. Besides, high SFRPs methylation showed favorable OS in GC patients. The functions of SFRPS were primarily related to the Wnt signaling pathway, immune system development, and basal cell carcinoma. The expression of SFRPS was strongly correlated with immune infiltrating cells, including CD4+ T cells and macrophages in GC. Our study indicated that SFRPS could be potential targets of precision therapy and prognostic biomarkers for the survival of GC patients.
\end{abstract}

Keywords: gastric cancer; SFRPs; prognostic value; immune infiltration; Wnt pathway

\section{Introduction}

Gastric cancer (GC) is the fifth most common malignant tumor and is the second leading cause of cancer-associated mortality worldwide according to the GLOBOCAN 2018 estimation [1]. Despite advances in the diagnosis and treatment of cancer, the prognosis of gastric cancer remains unsatisfactory due to the low diagnosis rate, with a 5-year overall survival lower than $40 \%$ [2]. Therefore, the exploration of a sensitive and specific biomarker that could predict the prognosis is crucial for GC management.

Secreted frizzled-related proteins (SFRPS) are extracellular tumor suppressor genes of Wnt signaling with roles in both embryogenesis and oncogenesis [3]. The loss of SFRP gene 
expression leading to downstream activation of the Wnt pathway is a vital mechanism for tumorigenesis [4]. In previous reports, frequent promoter hypermethylation and gene silencing of the SFRPS were identified in hepatocellular carcinoma and colorectal cancer [5]. Although SFRPs have demonstrated potential as effective biomarkers for some cancers, their roles in the development of other tumors are yet to be recognized.

SFRPS are modular proteins that contain the signal peptide for secretion followed by a cysteine-rich domain (CRD) [6]. Generally, SFRPs are thought to bind directly to Wnt ligands or Frizzled receptors, thereby preventing the initiation of the signaling cascades $[7,8]$. Some of SFRP genes and proteins have been characterized and studied by common expression profiles, and the aberrant expression of SFRPs has been reported to be associated with a variety of cancers [9]. However, the functions and prognostic values of different SFRP family members in GC remain unknown.

In the present study, we mined numerous large databases to analyze the expression, mutation, function, and immune infiltrating of SFRPs, with the aim of determining the potential oncogenic and prognostic values of distinct SFRPs in GC.

\section{Materials and Methods}

\subsection{Oncomine}

Oncomine (https:/ / www.oncomine.org/resource/login.html, accessed on 27 January 2021) is an integrated online database providing genome-wide expression analysis with cancer microarray information [10]. We used Oncomine database to analyze the expression levels of SFRPs family members in different types of cancer. The different mRNA expression levels between GC and normal tissues were analyzed with Student's $t$-test, with a threshold of $p$-value $<0.01$, fold change $\geq 2$, and top $10 \%$ gene rank.

\subsection{GEPIA}

GEPIA (http:/ / gepia.cancer-pku.cn/, accessed on 28 January 2021) [11] is an interactive web server for analyzing the RNA sequencing expression data from thousands of tumors and normal tissue samples. In our study, GEPIA was utilized to compare differential gene expression between GC and normal tissues. The pathological staging analysis and related prognostic analysis were also performed in GEPIA.

\subsection{UALCAN}

UALCAN (http:/ / ualcan.path.uab.edu/analysis.html, accessed on 26 May 2021) [12] was used to analyze the expression of 5 SFRPs genes between GC tissues and corresponding adjoining normal tissues. The difference in transcriptional levels was assessed by students' $t$-test considering unequal variance, and a $p$-value $<0.05$ was considered as statistically significant.

\subsection{Kaplan-Meier Plotter}

Kaplan-Meier plotter (http:/ /www.kmplot.com/, accessed on 27 May 2021) was used to evaluate the prognostic value of SFRPS mRNA expression in GC patients [13], which contained the association of gene expression data and survival information of patients with cancer. Hazard ratios (HR) with 95\% confidence intervals (CIs) and $p$-values were calculated and displayed in the survival charts, and the $p$-value $<0.05$ was considered as statistically significant.

\subsection{MethSurv}

MethSurv (https:/ / biit.cs.ut.ee/methsurv/, accessed on 16 April 2021) is a web tool to perform multivariable survival analysis using Cox proportional risk model, according to the TCGA database [14]. 


\section{6. cBioPortal}

cBioPortal (http:/ / www.cbioportal.org/, accessed on 29 January 2021) is a comprehensive web resource that provides visualization, analysis, and download of large-scale cancer genomics data sets [15]. In this study, five datasets, namely, "OncoSG 2018", "TCGA Firehose Legacy", "Pfizer and UHK Nat Genet 2014", "UHK Nat Genet 2011", and "U Tokyo Nat Genet 2014" were used for the analysis of SFRP gene mutations.

\subsection{STRING}

STRING (https://string-db.org/, accessed on 27 January 2021) [16] is an online database about predicting protein-protein interactions (10). Different expressions of five SFRPS and their possible interactions were collected and integrated through PPI network analysis.

\subsection{GeneMANIA}

GeneMANIA (http:/ /www.genemania.org, accessed on 27 January 2021) [17] was used to predict the protein and genetic interactions, pathways, and functions of five SFRP family members and their related interactors.

\subsection{DAVID}

Functions of SFRPS and 20 associated proteins were analyzed by Gene Ontology (GO) and Kyoto Encyclopedia of Genes and Genomes (KEGG) in the DAVID database (https: / / david.ncifcrf.gov/summary.jsp, accessed on 30 January 2021) [18,19]. GO enrichment analysis could predict the function of SFRPS and their 20 related proteins from biological processes (BP), cellular components (CC), and molecular functions (MF), while KEGG analysis could determine the related pathways of SFRPS and their associated interactors.

\subsection{TIMER}

TIMER (https: / cistrome.shinyapps.io/timer/, accessed on 26 May 2021) is a web resource for assessing the infiltration of different immune cells and their clinical impact [20]. SFRPS were input through the "Gene module" and generated plots, and the correlation between their expression and immune infiltration level in gastric cancer was observed.

Additional information of these databases are listed in Supplementary Table S1.

\section{Results}

\subsection{Differential mRNA Expression Levels of SFRPs in Patients with GC}

The transcriptional levels of five SFRPs were analyzed in 20 different types of human cancer and compared with normal individuals in the Oncomine database (Supplementary Figure S1). The mRNA expression levels of SFRP2 and SFRP4 were significantly elevated in the breast, gastric, and pancreatic cancer tissues, while SFRP1 showed significantly decreased expression in datasets from 15 different types of cancer. In the Forster Gastric dataset, SFRP4 over-expression was 8.758-fold higher $\left(p=4.90 \times 10^{-5}\right)$ in diffuse gastric adenocarcinoma tissues than in normal tissues, whereas Cho found a 3.437-fold increase $\left(p=7.77 \times 10^{-6}\right)$ and Chen found a 3.559-fold increase in SFRP4 mRNA expression $\left(p=1.93 \times 10^{-18}\right)$ in gastric intestinal-type adenocarcinoma tissues (Table 1$)$.

We then compared the mRNA expressions of SFRPs between GC and normal gastric tissues using the GEPIA dataset. The results showed that the expression levels of SFRP2 and SFRP4 in GC tissues were higher than those in normal tissues, and the expression levels of SFRP1 and SFRP5 were lower in gastric tissues than in normal tissues. These results were consistent with the findings from the UALCAN data set (Figure 1). 
Table 1. The significant changes of SFRPS expression in transcription level between gastric cancer and normal gastric tissues (Oncomine).

\begin{tabular}{cccccc}
\hline & Types of GC versus Normal & Fold Change & $\boldsymbol{p}$-Value & $\boldsymbol{t}$-Test & References \\
\hline \multirow{2}{*}{ SFRP1 } & Diffuse Gastric Adenocarcinoma & 2.488 & $4.79 \times 10^{-5}$ & 5.003 & Chen Gastric [21] \\
& Diffuse Gastric Adenocarcinoma & 12.858 & $3.47 \times 10^{-5}$ & 4.54 & Forster Gastric [22] \\
SFRP2 & Gastric Cancer & 9.956 & $1.78 \times 10^{-5}$ & 5.019 & Wang Gastric [23] \\
& Diffuse Gastric Adenocarcinoma & 10.073 & $7.00 \times 10^{-6}$ & 4.982 & Forster Gastric [22] \\
SFRP3 & Diffuse Gastric Adenocarcinoma & 5.896 & $3.95 \times 10^{-7}$ & 5.977 & Forster Gastric [22] \\
SFRP4 & Diffuse Gastric Adenocarcinoma & 4.814 & $5.75 \times 10^{-10}$ & 8.398 & Cho Gastric [24] \\
& Gastric Intestinal Type Adenocarcinoma & 3.437 & $7.77 \times 10^{-6}$ & 5.637 & Cho Gastric 2 [24] \\
& Diffuse Gastric Adenocarcinoma & 5.36 & $2.43 \times 10^{-6}$ & 7.716 & Chen Gastric [21] \\
& Gastric Intestinal Type Adenocarcinoma & 3.559 & $1.93 \times 10^{-18}$ & 11.327 & Chen Gastric 2 [21] \\
& Gastric Cancer & 3.423 & $3.74 \times 10^{-7}$ & 4.173 & Cui Gastric [25] \\
& Diffuse Gastric Adenocarcinoma & 8.758 & $4.90 \times 10^{-5}$ & 4.316 & Forster Gastric [22] \\
\hline
\end{tabular}

SFRP: secreted frizzled-related proteins, GC: gastric cancer, Student's $t$-test.

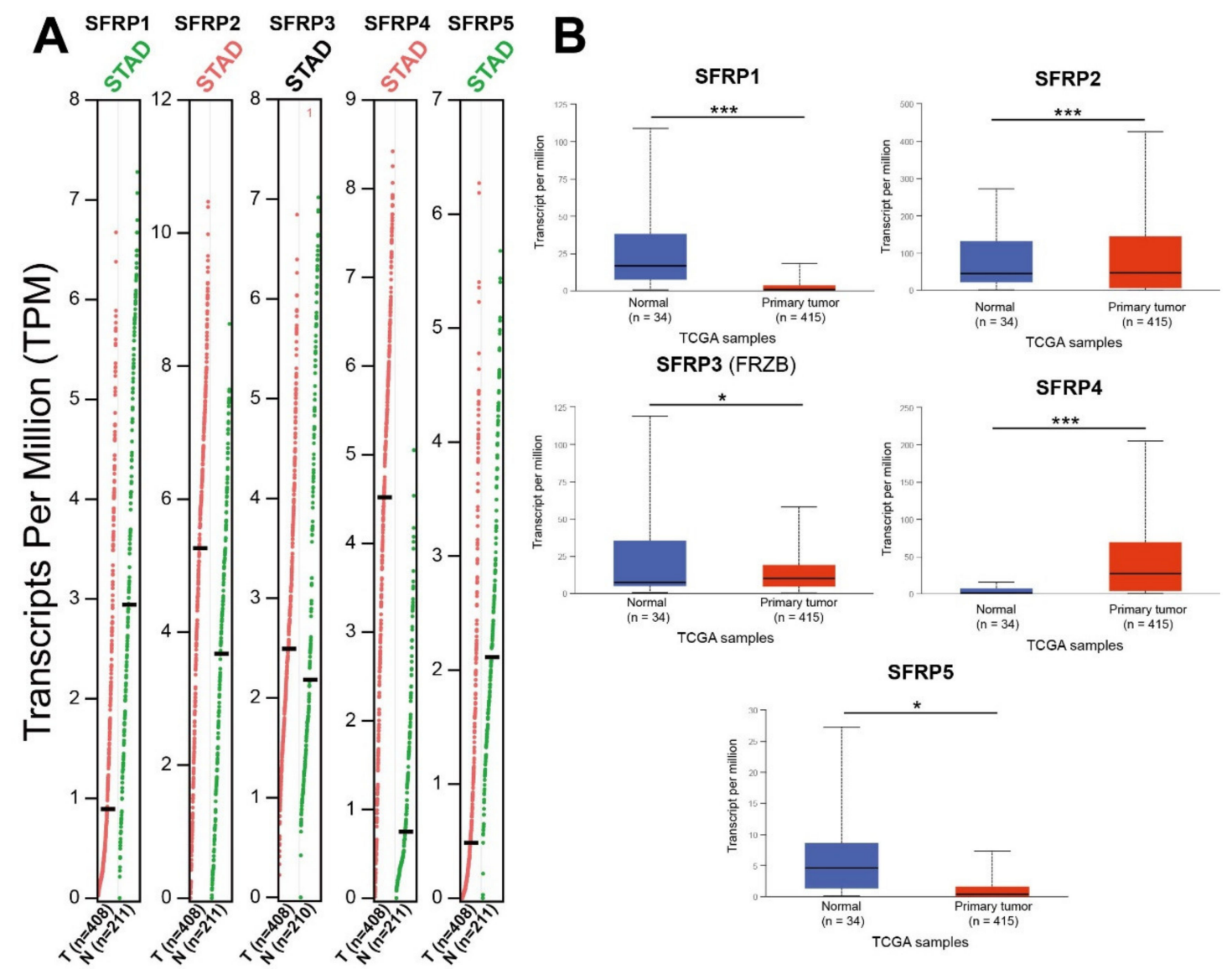

Figure 1. The mRNA expression of distinct SFRPS family members in GC tissues and normal gastric tissues (A: GEPIA database and B: UALCAN database). The method for differential analysis is t-test. ${ }^{*}: p<0.05$ and ${ }^{* * *}: p<0.001$.

\subsection{Relationship between SFRPS Expression Levels and Cancer Stages, Subtypes of GC Patients}

The expression of SFRPs in GC based on histological subtypes was also evaluated (Figure 2). We found that the expression of SFRP2/3/4 was higher in gastric adenocarcinoma of not otherwise specified (NOS) and diffuse type, and gastric intestinal adenocarcinoma of mucinous type. The expression of $S F R P 1 / 5$ was lower in gastric intestinal adenocarcinoma of NOS. We then evaluated the association between SFRPs expression and the pathological stage in patients with GC via GEPIA. The expression among tumor stages varied significantly for SFRP2, SFRP3, and SFRP4, whereas the mRNA expressions of SFRP1 and SFRP5 
were not markedly different (Supplementary Figure S2). We also analyzed the relationship between mRNA expressions of different SFRPS family members and individual cancer stages through UALCAN. The mRNA expressions of SFRP2/4 were the highest in GC stages 2, 3, and 4 . These results suggest that SFRP2, SFRP3, and SFRP4 might play an important role in the occurrence and development of GC.
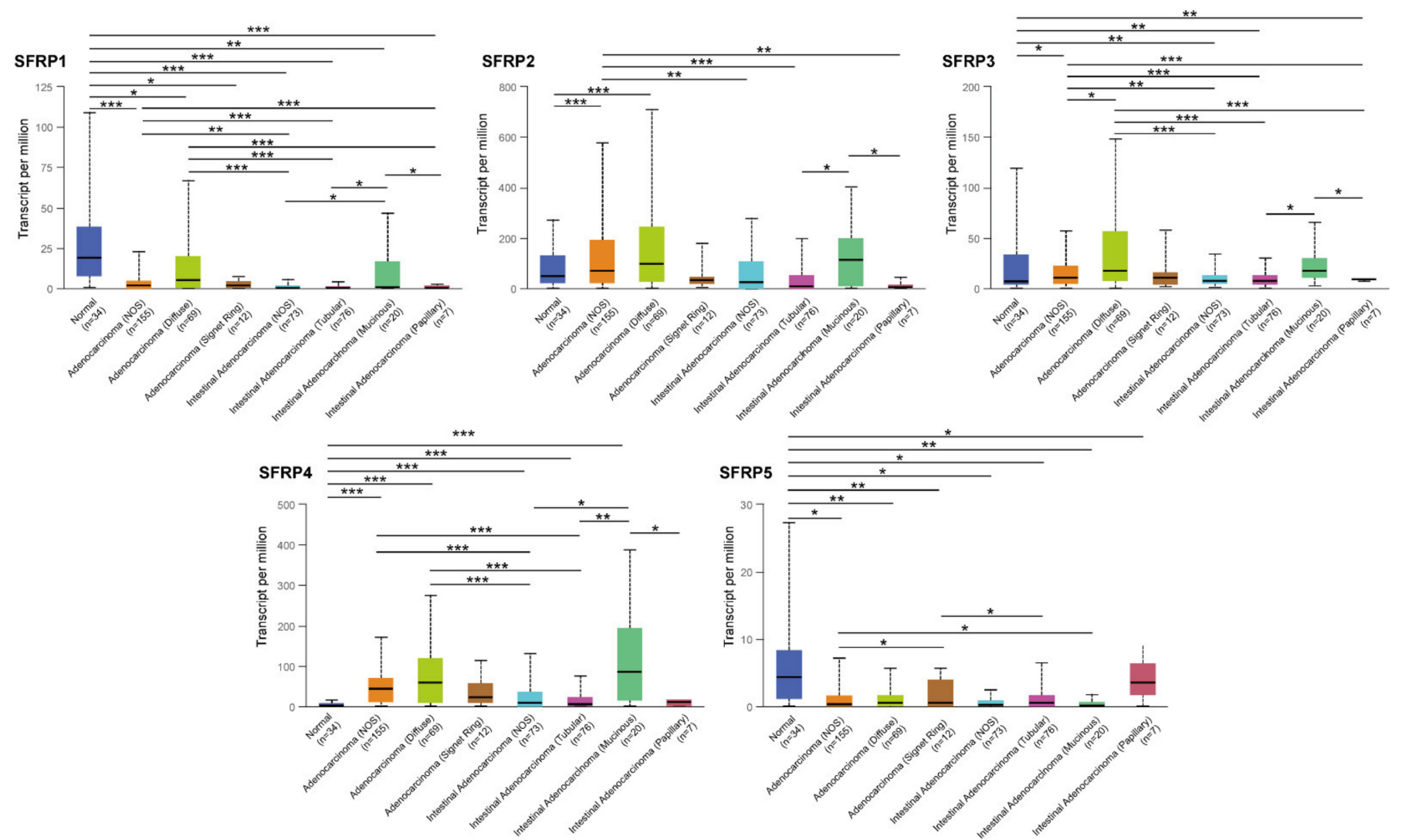

Figure 2. The SFRPs expression in different subtypes of GC (UALCAN database). The method for differential analysis is t-test. *: $p<0.05,{ }^{* *}: p<0.01$, and ${ }^{* * *}: p<0.001$. NOS: not otherwise specified.

The expression of SFRPs in GC based on tumor grade, nodal metastasis status, TP53 mutation status, and patient's age was also analyzed and exhibited in Supplementary Figures S3-S6. The differential expressions of SFRPS were also found in different subgroups of GC based on tumor grade, nodal metastasis status, and TP53 mutation status. Interestingly, the SFRP2/3/4 expressions were higher in patients between 41 and 60 years of age.

\subsection{Prognostic Value of SFRPs mRNA Expression in Patients with GC}

To evaluate the value of differential expression of SFRPs in GC progression, GEPIA was utilized to analyze the correlation between different SFRPS and clinical outcomes. The disease-free survival (DFS) and overall survival (OS) curves showed that GC patients with high transcriptional levels of SFRP1 ( $p=0.014)$, SFRP2 $(p=0.039)$, and SFRP5 $(p=0.038)$ were significantly associated with short DFS, but patients with high transcriptional levels of SFRP3 or SFRP4 did not show such association (Figure 3A). The Kaplan-Meier plotter was used to analyze the prognostic values of SFRPs in patients with GC (Figure 3B). The high mRNA expressions of every SFRP family member significantly correlated with short OS in patients with GC $(p<0.05)$. We further evaluated the prognostic values of SFRPs in subdivided GC patients based on stages of cancer, Lauren classification, type of treatments, and human epidermal growth factor 2 (HER2) receptor status (Supplementary Figures S7-S10). We found that only high expression of SFRP1 $(p<0.01)$ correlated with 
short OS in patients with all stages of GC. In addition, higher expression of SFRPs was associated with short OS in intestinal and mixed type GC patients. In surgery-treated and different HER2 status GC patients, the prognostic value of SFRPS mRNA expression was consistent.
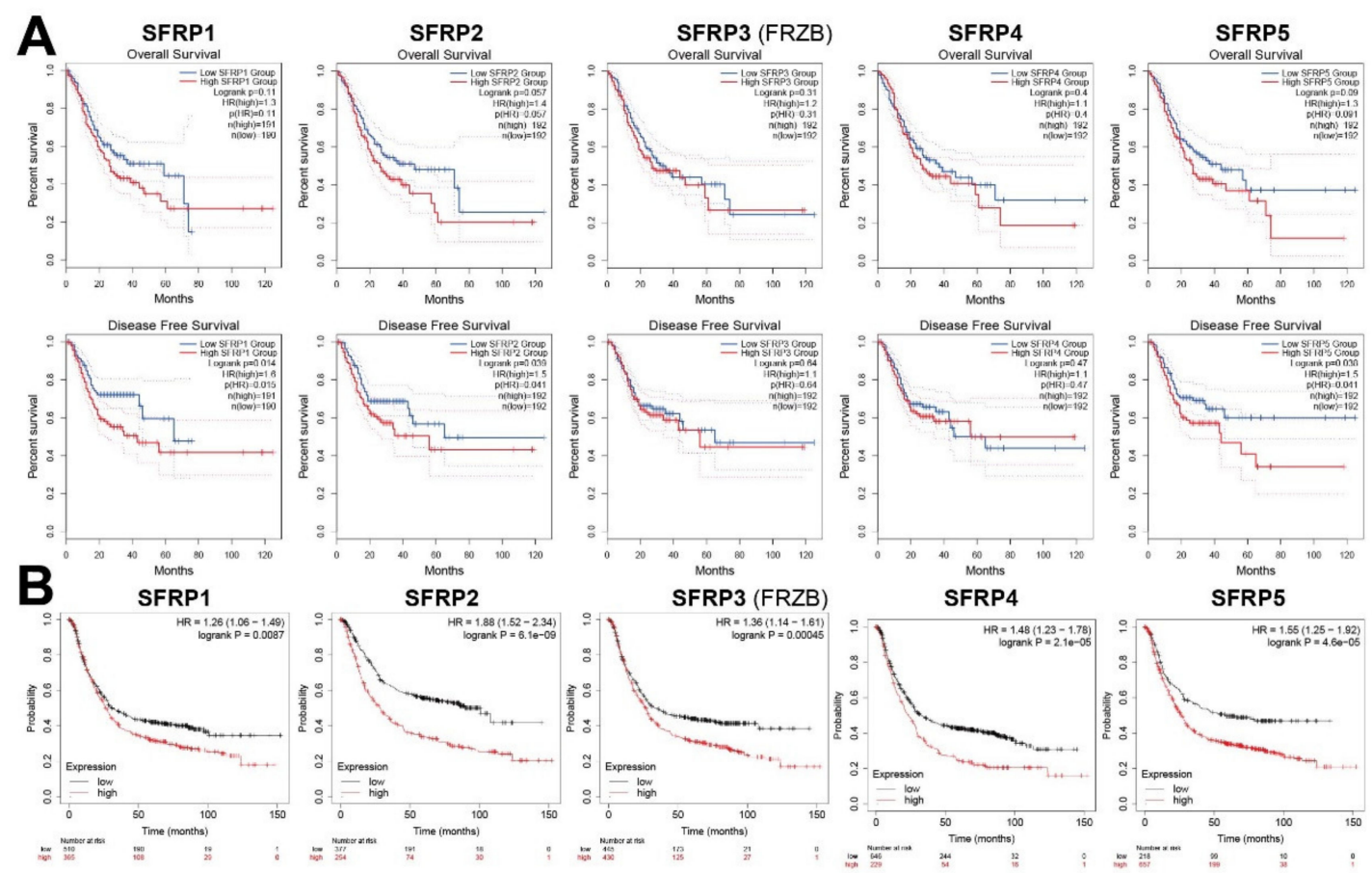

Figure 3. The prognostic value of mRNA level of SFRPS in patients with GC. (A) GEPIA database and (B) Kaplan-Meier plotter. The method for survival analysis is Log-rank test.

In addition, we selected the most relevant CpG sites $(|r|>0.5, p<0.01)$ to investigate the prognostic values of SFRPs methylation in patients with GC (Supplementary Figure S11). Kaplan-Meier plots demonstrated that low levels of SFRPs methylation of the selected CPG sites were correlated with short OS among patients with GC.

\subsection{Genetic Alteration and Interaction Analyses of SFRPs in Patients with GC}

Next, we used the cBioPortal online tool to analyze the genetic alterations of SFRPS in GC patients. We found that two or more alterations were detected in four subtypes of GC (Figure 4A). Among 777 GC patients sequenced, 80 GC patients had genetic alteration of SFRPs, with a mutation rate of $10 \%$. The mutation rates of SFRP1 and SFRP 4 were the highest, $3 \%$ and $5 \%$, respectively (Figure $4 \mathrm{~B}$ ).

The protein-protein interaction (PPI) network analysis was performed on the differentially expressed SFRPS and 10 proteins that significantly interacted with SFRPS using the STRING database to explore the potential interactions (Figure 4C). These differentially expressed SFRPS were associated with regulating the Wnt signaling pathway. The results from GeneMANIA also revealed that the function of differentially expressed SFRPs and their associated interactors (such as WNT4, FZD6, WNT2, FZD10, FZD2, FZD5, WNT8A, FZD3, FZD8, and DBNDD2) was primarily related to the Wnt signaling pathway, immune system development, and stem cell differentiation (Figure 4D).

\subsection{Go Enrichment and KEGG Pathway Analysis of SFRPs}

We used DAVID for Go enrichment and KEGG pathway analysis of SFRPs and their 20 interactors. The neuron differentiation and the Wnt signaling pathway were the main biological processes that were associated with target genes (Figure 5A). The proteinaceous extracellular matrix was the major cellular component of SFRPS and their interactors, and Wnt-activated receptor activity was their primary molecular function (Figure 5B,C). It was 
found that the Wnt signaling pathway, basal cell carcinoma, and melanogenesis of KEGG pathways for target genes were involved in GC (Figure 5D).

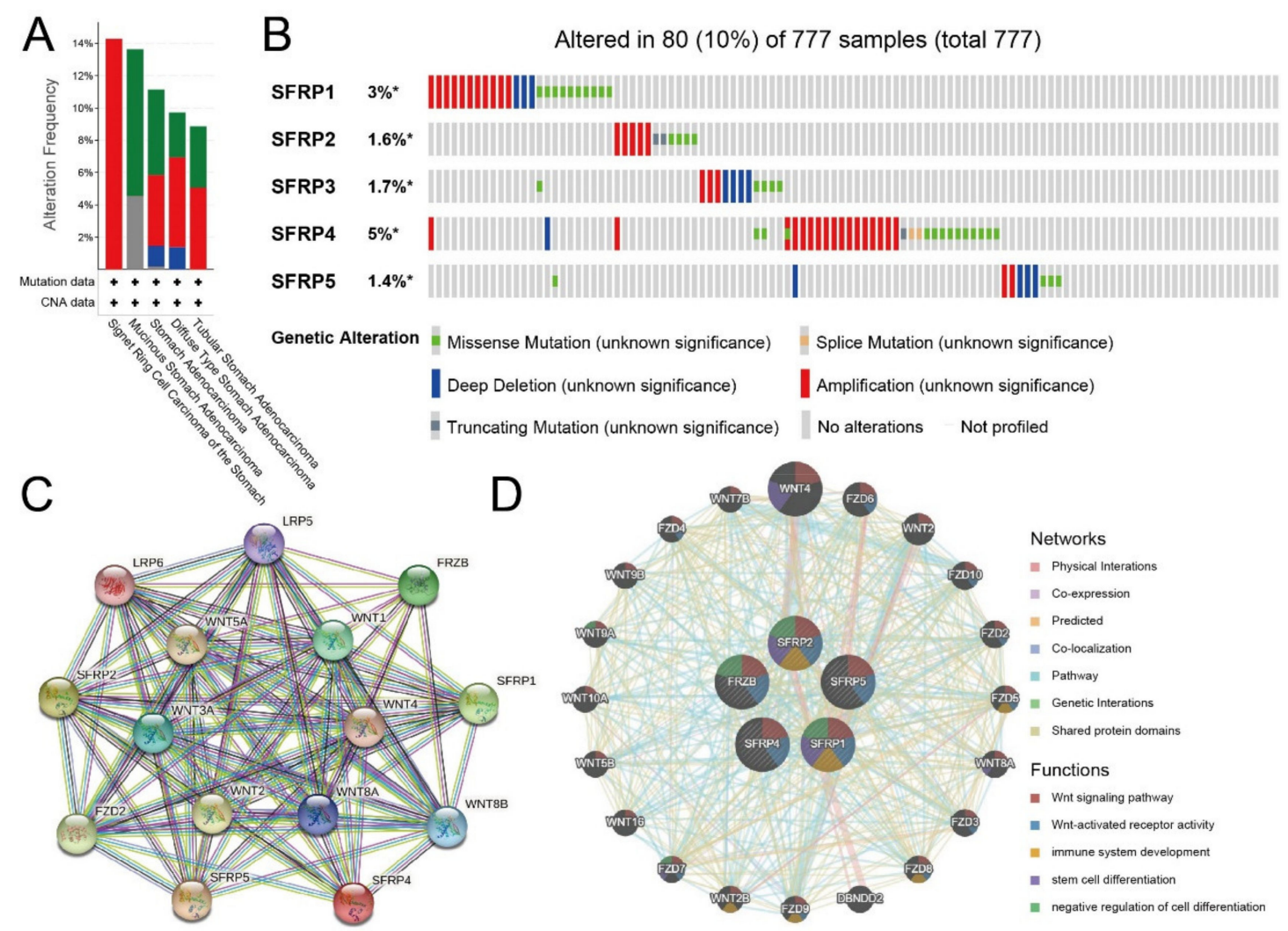

Figure 4. Gene mutation and expression analysis of SFRPs in patients with GC (cBioPortal and STRING). (A,B) Summary of genetic alterations in differently expressed SFRPs in GC. (C,D) Protein-protein interaction network of differently expressed SFRPS and their interactors using STRING and GeneMANIA databases, respectively.

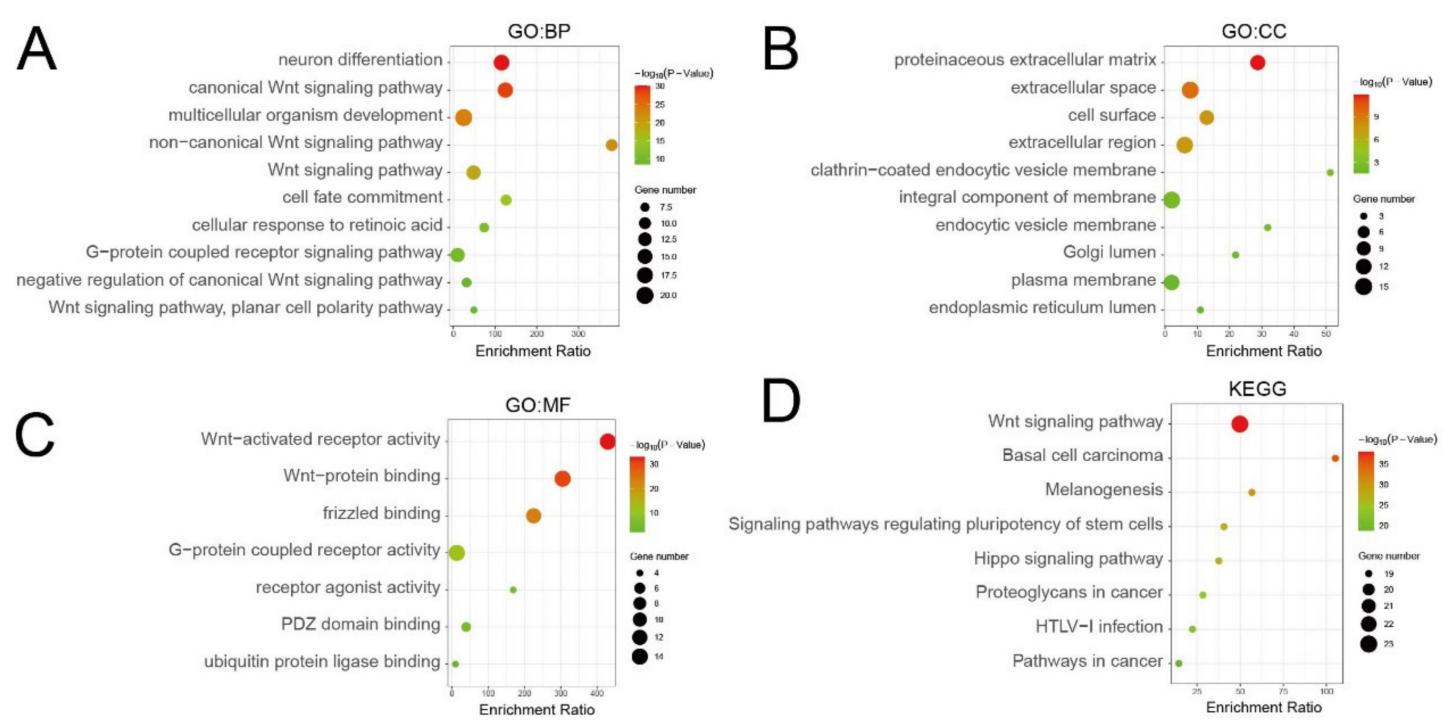

Figure 5. Gene Ontology (GO) enrichment and Kyoto Encyclopedia of Genes and Genomes (KEGG) pathway analysis of SFRPS and their interactors (DAVID). GO enrichment analysis of target genes based on (A) cellular component, (B) biological process, and (C) molecular function. (D) KEGG pathway enrichment analysis of target genes. 


\subsection{Immune Cell Infiltration of SFRPs in Patients with GC}

The TIMER database was utilized to investigate the association between SFRP family members and immune cell infiltration, as immune cell level correlates with the proliferation and progression of cancer cells (Figure 6). The expressions of $S F R P 1 / 3 / 4$ were positively correlated with the infiltration of CD8+ T cells $(p<0.001), \mathrm{CD} 4+\mathrm{T}$ cells $(p<0.001)$, macrophages $(p<0.001)$, neutrophils $(p<0.001)$, and dendritic cells $(p<0.001)$. SFRP2 was positively correlated with the infiltration of CD4+ T cells $(p<0.001)$, macrophages $(p<0.001)$, neutrophils $(p<0.001)$, and dendritic cells $(p<0.001)$. The SFRP5 expression was positively correlated with the infiltration of CD4+ T cells $(p<0.001)$ and macrophages $(p<0.001)$. In addition, the Cox proportional hazard model showed that CD4+ T cell $(p=0.04)$, macrophage $(p=0.013)$, and SFRP5 expression $(p=0.048)$ were significantly associated with clinical outcomes in GC patients (Table 2). The Cox proportional hazard model of SFRPs and clinical factors in GC was also evaluated (Supplementary Table S2). In addition, the results indicated that the separated SFRP1/2/4/5 expression, patients' age, and stage $3 / 4$ were significantly associated with clinical outcomes in GC.
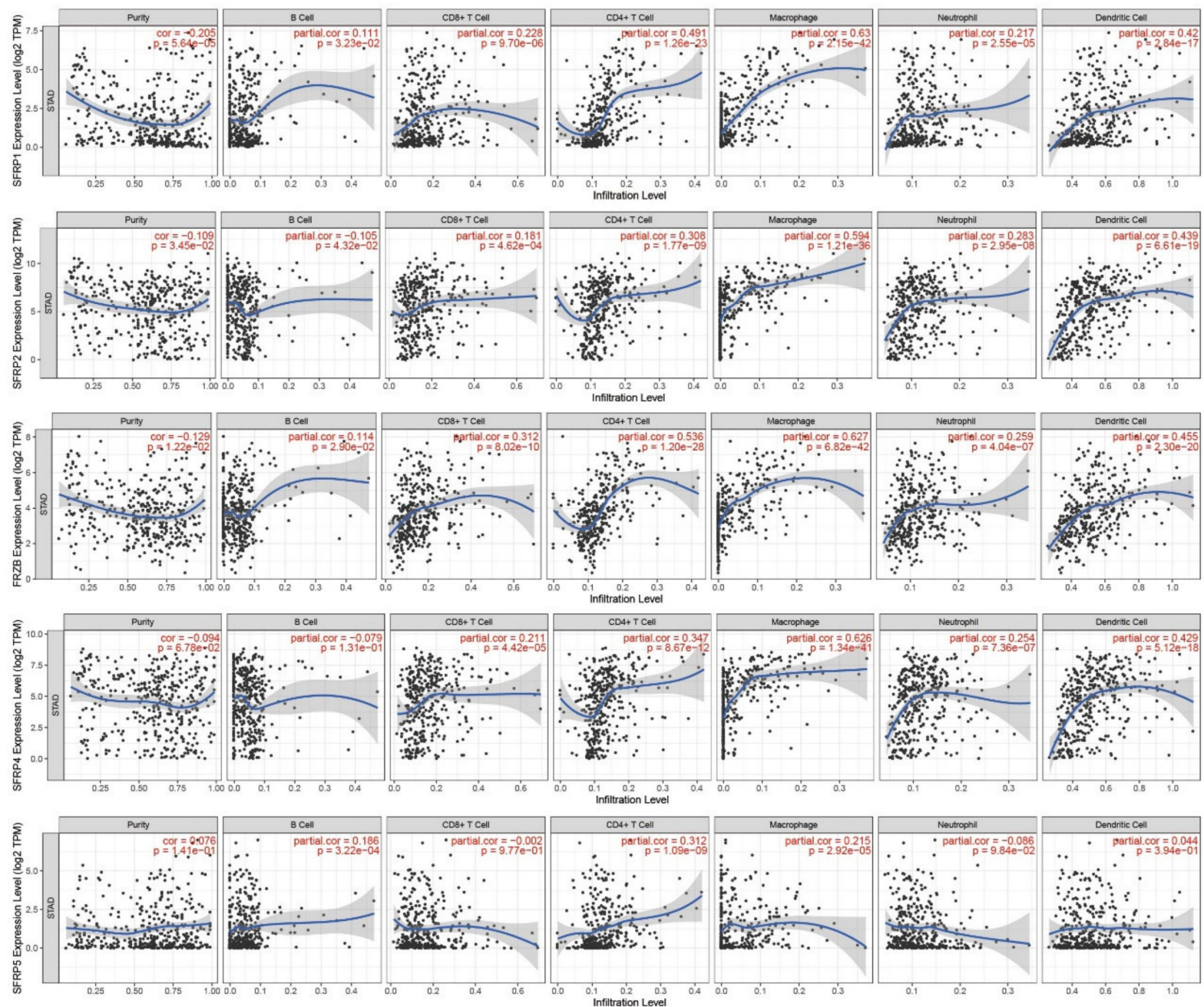

Figure 6. Correlations between differentially expressed SFRPS and immune cell infiltration (TIMER). Spearman's rho value was used for the analysis. 
Table 2. The Cox proportional hazard model of SFRPS and six tumor-infiltrating immune cells in GC (TIMER).

\begin{tabular}{ccccccc}
\hline & coef & HR & 95\% CI_1 & 95\% CI_u & $p$-Value & Sig. \\
\hline B cell & 4.041 & 56.862 & 0.936 & 3453.136 & 0.054 \\
CD8 T cell & -1.924 & 0.146 & 0.009 & 2.405 & 0.178 \\
CD4 T cell & -4.756 & 0.009 & 0 & 0.808 & 0.04 & $*$ \\
Macrophage & 4.655 & 105.108 & 2.667 & 4141.972 & 0.013 & $*$ \\
Neutrophil & 0.608 & 1.837 & 0.006 & 519.318 & 0.833 \\
Dendritic & 1.055 & 2.871 & 0.22 & 37.5 & 0.421 \\
SFRP1 & -0.057 & 0.945 & 0.802 & 1.112 & 0.493 \\
SFRP2 & 0.135 & 1.145 & 0.989 & 1.324 & 0.07 \\
SFRP3 & 0.076 & 1.079 & 0.901 & 1.292 & 0.41 \\
SFRP4 & -0.077 & 0.926 & 0.796 & 1.077 & 0.321 \\
SFRP5 & 0.112 & 1.118 & 1.001 & 1.249 & 0.048
\end{tabular}

Coef: coefficient, HR: hazard ratio, CI: confidence interval, sig: significance. ${ }^{*} p<0.05$.

Furthermore, we evaluated the association between SFRPS and several gene markers in GC using TIMER (Supplementary Figure S12). We found that the mRNA expressions of $S F R P S$ were positively correlated with the expression of FGFR1 $(p<0.001)$.

\section{Discussion}

Gastric cancer is the fifth most common malignant tumor and the second leading cause of cancer-associated mortality in the world. The pathogenesis of GC is a complex process, which is induced by numerous factors and further stimulated by a variety of pro-oncogenic pathways. The Wnt pathway is involved in important biological processes, such as cell proliferation and differentiation, and abnormal Wnt signaling is commonly observed in several types of cancer [26]. Secreted frizzled-related proteins (SFRPs), which are extracellular regulators and tumor suppressors, downregulate Wnt signaling by binding directly to Wnt ligands or Frizzled (Fz) receptors. Present studies have shown that SFRP methylation promotes carcinogenesis, especially in hepatocellular carcinoma and colorectal cancer. However, their exact function in GC remains to be elucidated [5]. In our study, we comprehensively analyzed SFRPs in terms of expression, mutation, prognostic value, functional enrichment, and immune cell infiltration.

We found that the expression of SFRP2 and SFRP4 in GC tissues was higher than that in normal tissues, while the expression of SFRP1 was decreased in GC tissues in the ONCOMINE database and UALCAN database. The expression of SFRP5 was also significantly reduced in GC tissues in GEPIA and UALCAN. The expression of SFRP2, $S F R P 3$, and SFRP4 in patients with GC significantly correlated with the clinical tumor stage. In addition, the expression of SFRP2/3/4 was higher in gastric adenocarcinoma of NOS and diffuse type, and gastric intestinal adenocarcinoma of mucinous type. The expression of $S F R P 1 / 5$ was lower in gastric intestinal adenocarcinoma of NOS. In addition, the mRNA expressions of SFRP2/4 were the highest in GC stage 2/3/4, and the SFRP2/3/4 expressions were higher in patients between 41 and 60 years of age.

Overexpression of SFRP1, SFRP2, and SFRP5 in GC significantly correlated with short DFS. Higher expression of SFRP1/3 correlated with short OS in patients with all stages of GC. We also identified the prognostic values of SFRPS in subdivided GC patients based on stages of cancer, Lauren classification, type of treatments, and HER2 status. The higher expression of SFRPS was associated with short OS in intestinal and mixed type GC patients, as well as in surgery-treated and different HER2 status GC patients. These results suggest that SFRPS are involved in the tumorigenesis of GC and carry potential as a prognostic biomarker for GC.

SFRP2 has previously been reported as an anti-oncogene whose methylation has been shown to accelerate cancer cell invasion and growth during tumor progression [27]. SFRP2 can compete with Fz receptors to interact with Wnt proteins via its frizzled-like CRD [6]. A previous study demonstrated that the overexpression of SFRP2 inhibits the 
proliferation of oral squamous carcinoma cells and blocks the cell cycle in the G1 phase [28]. The levels of SFRP1, SFRP2, and SFRP5 methylation were also reported up-regulated in hepatocellular carcinoma tissues [29]. Previous studies found that the methylation levels of SFRP2 in gastric carcinoma were lower than adjacent non-cancer samples, and overexpression of SFRP2 in vivo can inhibit the proliferation of tumor cell and induce cell apoptosis, demonstrated that the methylation of $S F R P 2$ is an early event in the process of GC [27]. As a result, SFRP2 can be a novel biomarker and a potential drug target of GC.

Other SFRPS are aberrantly expressed in tumors, regulate tumorigenesis, and may serve as potential prognostic biomarkers in GC. SFRP1 and SFRP2 have shown oncogenic potential by increasing cellular proliferation or invasion and promoting in vivo tumor growth in renal cancer [30,31]. SFRP1 was also reported to inhibit several cancers, which was mainly due to epigenetic inactivation via DNA methylation or transcriptional silencing by microRNAs. Epigenetic silencing of SFRP1 may cause dysregulation of cell proliferation, migration, and invasion [32]. We found that SFRP1 is significantly reduced in GC tissues and associated with short DFS, thereby warranting further exploration of its functions. The methylation of SFRP3 promoter was reported frequently in hepatocellular carcinoma [33]. In our study, the expression of SFRP3 was significantly correlated with gastric cancer stages.

SFRP4 is a relatively novel Wnt antagonist, which has garnered considerable attention in recent years due to its regulatory action in the Wnt signal transduction system [34]. SFRP4 is involved in cell proliferation and differentiation and plays an important role in carcinogenesis [35]. Consistent with current literature, we found that the expression of SFRP4 and gastric cancer stages were positively correlated. A previous finding confirmed the role of SFRP5 as a physiologic tumor suppressor and demonstrated its potential diagnostic and prognostic value in CRC. We demonstrated that high SFRP5 expression was significantly correlated with short DFS and OS in GC. Besides, the high SFRPs methylation showed favorable OS in GC patients.

In addition, the interaction network and enrichment analysis demonstrated that SFRPS and their 20 interactors were mainly associated with the Wnt signaling pathway, immune system development, and basal cell carcinoma. Previous studies showed that SFRP4 correlates with Treg cell infiltration in pancreatic ductal adenocarcinoma [36]. We further explored the relationship of SFRPs and tumor-infiltrating immune cells in GC and found that the expression of SFRPS was positively correlated with the infiltration of $\mathrm{CD} 4+\mathrm{T}$ cells and macrophages. The Cox proportional hazard model indicated that the separated SFRP $1 / 2 / 4 / 5$ expression, CD4+ T cell, macrophage, patients' age, and stage 3/4 were significantly associated with clinical outcomes in GC. In addition, the expression of SFRPS was positively correlated with the expression of FGFR1, which was an independent prognostic factor in gastric cancer [37]. These findings suggest that SFRPs may play a significant role in the tumor microenvironment.

Our study has some limitations that need to be addressed. All the data analyzed in our study came from online databases, and a larger cohort is needed to validate our findings and explore the clinical application of the SFRPs members in the GC therapy. In addition, we did not explore the potential mechanisms of distinct SFRPs in GC.

\section{Conclusions}

This comprehensive bioinformatics analysis investigated the mRNA expression patterns, prognostic values, genetic alterations, PPI network, functional enrichment, and immune infiltration of $S F R P$ s in patients with GC. Our results revealed that $S F R P 1,2$, and 5 may be new prognostic biomarkers and SFRP2 2, 3, and 4 may be potential targets for GC. In addition, the high levels of SFRPs methylation were associated with better OS among patients with GC. The expression of SFRPS correlated significantly with the infiltration of CD4+ T cells and macrophages and the expression of FGFR1. Finally, these findings would contribute to novel insights into the distinct roles of SFRPs in GC and make a strong argument for further investigation into the application of SFRP in GC management. 
Supplementary Materials: The following are available online at https: / www.mdpi.com/article/ 10.3390/life11060522/s1, Figure S1: The mRNA expression of SFRPS in different cancer types (Oncomine). Difference of transcriptional expression was compared by Students' $t$-test, Figure S2: Correlations between SFRPs expression and tumor stage in GC patients (A: GEPIA database, B: UALCAN database). One-way ANOVA was conducted for differential gene expression analysis using pathological stage as a variable and $\log 2(\mathrm{TPM}+1)$ transformed expression data were used for plotting in GEPIA, Figure S3: The SFRPS expression in GC based on tumor grade (UALCAN database). The method for differential analysis is $t$ test. ${ }^{*}: p<0.05,{ }^{* *}: p<0.01$ and ${ }^{* * *}: p<0.001$. Grade 1: well differentiated, Grade 2: moderately differentiated, Grade 3: poorly differentiated, Grade 4: undifferentiated, Figure S4: The SFRPs expression in GC based on nodal metastasis status (UALCAN database). The method for differential analysis is $t$ test. ${ }^{*}: p<0.05,{ }^{* *}: p<0.01$, and ***: $p<0.001$. N0: no regional lymph node metastasis, N1: metastases in 1-3 axillary lymph nodes, N2: metastases in 4-9 axillary lymph nodes, N3: metastases in 10 or more axillary lymph nodes, Figure S5: The SFRPS expression in GC based on TP53 mutation status (UALCAN database). The method for differential analysis is $t$ test. ${ }^{*}: p<0.05,{ }^{* *}: p<0.01$, and ${ }^{* * *}: p<0.001$, Figure S6: The SFRPS expression in GC based on the ages of patients (UALCAN database). The method for differential analysis is $t$ test. ${ }^{*}: p<0.05,{ }^{* *}: p<0.01$, and ${ }^{* * *}: p<0.001$. Yrs: years, Figure S7: The prognostic value of mRNA level of SFRPs in patients with different GC stages (Kaplan-Meier plotter). The method for survival analysis is Log-rank test, Figure S8: The prognostic value of mRNA level of SFRPS in patients with different GC subtypes (Kaplan-Meier plotter). A: intestinal type gastric carcinoma, B: diffuse type gastric carcinoma, $C$ : mixed type gastric carcinoma. The method for survival analysis is Log-rank test, Figure S9: The prognostic value of mRNA level of SFRPS in GC patients with different treatment (Kaplan-Meier plotter). A: surgery alone, B: 5 FU-based adjuvant therapy. The method for survival analysis is Log-rank test, Figure S10: The prognostic value of mRNA level of SFRPS in GC patients with different HER2 status (Kaplan-Meier plotter). A: HER2 negative and B: HER2 positive. The method for survival analysis is Log-rank test, Figure S11: Kaplan-Meier curves of low and high SFRPS DNA promoter CpG sites in GC patients (A: SFRP1, B: SFRP2, C: SFRP3, D: SFRP4, and E: SFRP5). The method for survival analysis is Log-rank test, Figure S12: Correlations between differentially expressed SFRPS and usual gene markers in GC (TIMER). Spearman's rho value was used for the analysis, Table S1: The information of database, Table S2: The Cox proportional hazard model of SFRPs and clinical factors (TIMER).

Author Contributions: Conceptualization, D.L. and K.Z.; data curation, D.L.; formal analysis, C.S., N.K., and Y.C.; funding acquisition, K.Z.; methodology, D.L.; project administration, K.Z.; resources, D.L.; software, D.L.; supervision, K.Z.; validation, D.L. and K.Z.; visualization, D.L.; writing-original draft, D.L.; writing-review and editing, C.S., N.K., C.B., J.P.W.T., Y.C., S.M., Y.H., C.C., Q.Z., and K.Z. All authors have read and agreed to the published version of the manuscript.

Funding: This research was funded by the Anhui Science and Technology Department, grant number 1604a0802075.

Institutional Review Board Statement: Not applicable.

Informed Consent Statement: Not applicable.

Data Availability Statement: The data comes from Oncomine, GEPIA, UALCAN, Kaplan-Meier plotter, cBioPortal, STRING, Gene-MANIA, DAVID, MethSurv, and TIMER databases.

Conflicts of Interest: The authors declare no conflict of interest.

\section{References}

1. Bray, F.; Ferlay, J.; Soerjomataram, I.; Siegel, R.L.; Torre, L.A.; Jemal, A. Global cancer statistics 2018: GLOBOCAN estimates of incidence and mortality worldwide for 36 cancers in 185 countries. CA Cancer J. Clin. 2018, 68, 394-424. [CrossRef]

2. Tan, Z. Recent Advances in the Surgical Treatment of Advanced Gastric Cancer: A Review. Med. Sci. Monit. 2019, 25, 3537-3541. [CrossRef]

3. Shi, Y.; He, B.; You, L.; Jablons, D.M. Roles of secreted frizzled-related proteins in cancer. Acta Pharmacol. Sin. 2007, 28, 1499-1504. [CrossRef]

4. Yin, P.; Wang, W.; Zhang, Z.; Bai, Y.; Gao, J.; Zhao, C. Wnt signaling in human and mouse breast cancer: Focusing on Wnt ligands, receptors and antagonists. Cancer Sci. 2018, 109, 3368-3375. [CrossRef] [PubMed] 
5. Yu, J.; Xie, Y.; Li, M.; Zhou, F.; Zhong, Z.; Liu, Y.; Wang, F.; Qi, J. Association between SFRP promoter hypermethylation and different types of cancer: A systematic review and meta-analysis. Oncol. Lett. 2019, 18, 3481-3492. [CrossRef] [PubMed]

6. Rattner, A.; Hsieh, J.C.; Smallwood, P.M.; Gilbert, D.J.; Copeland, N.G.; Jenkins, N.A.; Nathans, J. A family of secreted proteins contains homology to the cysteine-rich ligand-binding domain of frizzled receptors. Proc. Natl. Acad. Sci. USA 1997, 94, $2859-2863$. [CrossRef] [PubMed]

7. Galli, L.M.; Barnes, T.; Cheng, T.; Acosta, L.; Anglade, A.; Willert, K.; Nusse, R.; Burrus, L.W. Differential inhibition of Wnt-3a by Sfrp-1, Sfrp-2, and Sfrp-3. Dev. Dyn. 2006, 235, 681-690. [CrossRef] [PubMed]

8. Martin-Manso, G.; Calzada, M.J.; Chuman, Y.; Sipes, J.M.; Xavier, C.P.; Wolf, V.; Kuznetsova, S.A.; Rubin, J.S.; Roberts, D.D. sFRP-1 binds via its netrin-related motif to the N-module of thrombospondin-1 and blocks thrombospondin-1 stimulation of MDA-MB-231 breast carcinoma cell adhesion and migration. Arch Biochem. Biophys. 2011, 509, 147-156. [CrossRef]

9. Surana, R.; Sikka, S.; Cai, W.; Shin, E.M.; Warrier, S.R.; Tan, H.J.; Arfuso, F.; Fox, S.A.; Dharmarajan, A.M.; Kumar, A.P. Secreted frizzled related proteins: Implications in cancers. Biochim. Biophys. Acta 2014, 1845, 53-65. [CrossRef]

10. Rhodes, D.R.; Yu, J.; Shanker, K.; Deshpande, N.; Varambally, R.; Ghosh, D.; Barrette, T.; Pandey, A.; Chinnaiyan, A.M. ONCOMINE: A cancer microarray database and integrated data-mining platform. Neoplasia 2004, 6, 1-6. [CrossRef]

11. Tang, Z.; Li, C.; Kang, B.; Gao, G.; Li, C.; Zhang, Z. GEPIA: A web server for cancer and normal gene expression profiling and interactive analyses. Nucleic Acids Res. 2017, 45, W98-W102. [CrossRef] [PubMed]

12. Chandrashekar, D.S.; Bashel, B.; Balasubramanya, S.A.H.; Creighton, C.J.; Ponce-Rodriguez, I.; Chakravarthi, B.; Varambally, S. UALCAN: A Portal for Facilitating Tumor Subgroup Gene Expression and Survival Analyses. Neoplasia 2017, 19, 649-658. [CrossRef] [PubMed]

13. Gyorffy, B.; Surowiak, P.; Budczies, J.; Lanczky, A. Online Survival Analysis Software to Assess the Prognostic Value of Biomarkers Using Transcriptomic Data in Non-Small-Cell Lung Cancer. PLoS ONE 2014, 9, e82241. [CrossRef] [PubMed]

14. Modhukur, V.; Iljasenko, T.; Metsalu, T.; Lokk, K.; Laisk-Podar, T.; Vilo, J. MethSurv: A web tool to perform multivariable survival analysis using DNA methylation data. Epigenomics 2018, 10, 277-288. [CrossRef]

15. Gao, J.J.; Aksoy, B.A.; Dogrusoz, U.; Dresdner, G.; Gross, B.; Sumer, S.O.; Sun, Y.C.; Jacobsen, A.; Sinha, R.; Larsson, E.; et al. Integrative Analysis of Complex Cancer Genomics and Clinical Profiles Using the cBioPortal. Sci. Signal 2013, 6, 11. [CrossRef]

16. Szklarczyk, D.; Gable, A.L.; Lyon, D.; Junge, A.; Wyder, S.; Huerta-Cepas, J.; Simonovic, M.; Doncheva, N.T.; Morris, J.H.; Bork, P.; et al. STRING v11: Protein-protein association networks with increased coverage, supporting functional discovery in genome-wide experimental datasets. Nucleic Acids Res. 2019, 47, D607-D613. [CrossRef]

17. Vlasblom, J.; Zuberi, K.; Rodriguez, H.; Arnold, R.; Gagarinova, A.; Deineko, V.; Kumar, A.; Leung, E.; Rizzolo, K.; Samanfar, B.; et al. Novel function discovery with GeneMANIA: A new integrated resource for gene function prediction in Escherichia coli. Bioinformatics 2015, 31, 306-310. [CrossRef]

18. Huang, D.W.; Sherman, B.T.; Lempicki, R.A. Systematic and integrative analysis of large gene lists using DAVID bioinformatics resources. Nat. Protoc. 2009, 4, 44-57. [CrossRef]

19. Huang, D.W.; Sherman, B.T.; Lempicki, R.A. Bioinformatics enrichment tools: Paths toward the comprehensive functional analysis of large gene lists. Nucleic Acids Res. 2009, 37, 1-13. [CrossRef]

20. Li, T.W.; Fan, J.Y.; Wang, B.B.; Traugh, N.; Chen, Q.M.; Liu, J.S.; Li, B.; Liu, X.S. TIMER: A Web Server for Comprehensive Analysis of Tumor-Infiltrating Immune Cells. Cancer Res. 2017, 77, E108-E110. [CrossRef]

21. Chen, X.; Leung, S.Y.; Yuen, S.T.; Chu, K.M.; Ji, J.F.; Li, R.; Chan, A.S.Y.; Law, S.; Troyanskaya, O.G.; Wong, J.; et al. Variation in gene expression patterns in human gastric cancers. Mol. Biol. Cell 2003, 14, 3208-3215. [CrossRef]

22. Forster, S.; Gretschel, S.; Jons, T.; Yashiro, M.; Kemmner, W. THBS4, a novel stromal molecule of diffuse-type gastric adenocarcinomas, identified by transcriptome-wide expression profiling. Modern Pathol. 2011, 24, 1390-1403. [CrossRef]

23. Wang, Q.; Wen, Y.G.; Li, D.P.; Xia, J.; Zhou, C.Z.; Yan, D.W.; Tang, H.M.; Peng, Z.H. Upregulated INHBA expression is associated with poor survival in gastric cancer. Med. Oncol. 2012, 29, 77-83. [CrossRef]

24. Cho, J.Y.; Lim, J.Y.; Cheong, J.H.; Park, Y.Y.; Yoon, S.L.; Kim, S.M.; Kim, S.B.; Kim, H.; Hong, S.W.; Park, Y.N.; et al. Gene Expression Signature-Based Prognostic Risk Score in Gastric Cancer. Clin. Cancer Res. 2011, 17, 1850-1857. [CrossRef] [PubMed]

25. Cui, J.A.; Chen, Y.B.; Chou, W.C.; Sun, L.K.; Chen, L.; Suo, J.A.; Ni, Z.H.; Zhang, M.; Kong, X.X.; Hoffman, L.L.; et al. An integrated transcriptomic and computational analysis for biomarker identification in gastric cancer. Nucleic Acids Res. 2011, 39, 1197-1207. [CrossRef] [PubMed]

26. De Oliveira, L.A.; Oshima, C.T.F.; Soffner, P.A.; Silva, M.D.; Lins, R.R.; Malinverni, A.C.D.; Waisberg, J. The Canonical Wnt Pathway in Gastric Carcinoma. ABCD Arq. Bras. Cir. Dig. 2019, 32, e1414. [CrossRef] [PubMed]

27. Liu, Y.; Zhou, Q.; Zhou, D.; Huang, C.; Meng, X.; Li, J. Secreted frizzled-related protein 2-mediated cancer events: Friend or foe? Pharmacol. Rep. 2017, 69, 403-408. [CrossRef] [PubMed]

28. Xiao, C.; Wang, L.; Zhu, L.; Zhang, C.; Zhou, J. Secreted frizzledrelated protein 2 is epigenetically silenced and functions as a tumor suppressor in oral squamous cell carcinoma. Mol. Med. Rep. 2014, 10, 2293-2298. [CrossRef] [PubMed]

29. Lin, H.H.; Feng, W.C.; Lu, L.C.; Shao, Y.Y.; Hsu, C.H.; Cheng, A.L. Inhibition of the Wnt/beta-catenin signaling pathway improves the anti-tumor effects of sorafenib against hepatocellular carcinoma. Cancer Lett. 2016, 381, 58-66. [CrossRef]

30. Saini, S.; Liu, J.; Yamamura, S.; Majid, S.; Kawakami, K.; Hirata, H.; Dahiya, R. Functional significance of secreted Frizzled-related protein 1 in metastatic renal cell carcinomas. Cancer Res. 2009, 69, 6815-6822. [CrossRef] [PubMed] 
31. Yamamura, S.; Kawakami, K.; Hirata, H.; Ueno, K.; Saini, S.; Majid, S.; Dahiya, R. Oncogenic functions of secreted Frizzled-related protein 2 in human renal cancer. Mol. Cancer Ther. 2010, 9, 1680-1687. [CrossRef] [PubMed]

32. Baharudin, R.; Tieng, F.Y.F.; Lee, L.H.; Ab Mutalib, N.S. Epigenetics of SFRP1: The Dual Roles in Human Cancers. Cancers 2020, 12, 445. [CrossRef] [PubMed]

33. Lin, Y.W.; Shih, Y.L.; Lien, G.S.; Suk, F.M.; Hsieh, C.B.; Yan, M.D. Promoter methylation of SFRP3 is frequent in hepatocellular carcinoma. Dis. Markers 2014, 2014, 351863. [CrossRef]

34. Pawar, N.M.; Rao, P. Secreted frizzled related protein 4 (sFRP4) update: A brief review. Cell Signal 2018, 45, 63-70. [CrossRef]

35. Pohl, S.; Scott, R.; Arfuso, F.; Perumal, V.; Dharmarajan, A. Secreted frizzled-related protein 4 and its implications in cancer and apoptosis. Tumor Biol. 2015, 36, 143-152. [CrossRef] [PubMed]

36. Yang, M.W.; Tao, L.Y.; Yang, J.Y.; Jiang, Y.S.; Fu, X.L.; Liu, W.; Huo, Y.M.; Li, J.; Zhang, J.F.; Hua, R.; et al. SFRP4 is a prognostic marker and correlated with Treg cell infiltration in pancreatic ductal adenocarcinoma. Am. J. Cancer Res. $2019,9,363-377$.

37. Xie, G.; Ke, Q.; Ji, Y.Z.; Wang, A.Q.; Jing, M.; Zou, L.L. FGFR1 is an independent prognostic factor and can be regulated by miR-497 in gastric cancer progression. Braz. J. Med. Biol. Res. 2019, 52, e7816. [CrossRef] [PubMed] 